\title{
Loss-of-function ferroportin disease: novel mechanistic insights and unanswered questions
}

\section{Tom Vlasveld ${ }^{1}$ and Dorine W. Swinkels ${ }^{2}$}

\author{
${ }^{1}$ Department of Internal Medicine, Haaglanden Medical Center, Location Bronovo, The Hague and ${ }^{2}$ Translational Metabolic Laboratory, \\ Department of Laboratory Medicine, Radboud University Medical Center, Nijmegen, the Netherlands
}

E-mail:dorine.swinkels@radboudumc.nl

doi:10.3324/haematol.2018.203315

F erroportin, a 571 amino acid cation transporter encoded by the SLC40A1 gene, is the only known human cellular iron exporter and primarily expressed in the basolateral membrane of duodenal enterocytes, macrophages, and hepatocytes. ${ }^{1,2}$ By regulating ferroportin-mediated iron export in these cells, the hepatocyte-derived peptide hormone hepcidin plays a central role in intestinal iron resorption, macrophage iron recycling and hepatic iron storage (Figure 1A). ${ }^{3}$ Functional studies reveal the internalization of ferroportin upon hepcidin binding with subsequent ubiquitination of the protein resulting in the diminution of cellular iron export. The mechanism of the inhibition of cellular iron efflux is not fully elucidated. ${ }^{4}$ Genetic ferroportin variants result in autosomal dominantly inherited hereditary hemochromatosis $(\mathrm{HH})$ type 4 or ferroportin disease which is traditionally divided into two entities primarily based on the pattern of cellular iron distribution. Classical ferroportin disease (type 4A) is characterized by macrophage iron retention and decreased circulating iron availability for erythropoiesis, and is clinically recognized by the presence of elevated serum ferritin concentrations with low to normal transferrin saturation (TSAT) and poor tolerance to phlebotomy. Non-classical (atypical, type 4B or ferroportinassociated $\mathrm{HH}$ ) ferroportin disease is characterized by parenchymal (hepatocellular) iron overload with both elevated serum ferritin and TSAT and is indistinguishable from other forms of $\mathrm{HH}^{5}$

To assess the pathogenicity of a ferroportin variant functional studies on iron export and/or ferroportin expression are performed in cell-lines (mostly HEK293T, a human embryonic kidney cell line) transfected with the variant. However, interpretation of results may be hampered by these studies being done in non-enterocyte and non-macrophage cell lines. Moreover, comparability between reports may have shortcomings, since iron loading and hepcidin exposure protocols, the mode of iron export measurements and the determination of ferroportin expression differ largely between studies. Nevertheless, the results obtained are quite consistent, in that patients with the type 4A phenotype are mostly associated with loss-of-function ferroportin variants which display decreased membrane expression and/or iron transport in functional assays, while patients with the type $4 \mathrm{~B}$ phenotype have gain-of-function variants with preserved membrane expression and iron export capacity even after hepcidin exposure. Notwithstanding a different pattern of cellular iron distribution, a review of numerous case series of ferroportin disease reveals that in those patients who underwent magnetic resonance imaging (MRI) or liver biopsy, hepatic iron content is increased for both types of ferroportin disease. These observations indicate that loss-of-function variants also result in body iron overload.

Variety in iron parameters reported for patients with ferroportin variants is large and the correlation between iron export capacity in functional studies and clinical phenotype is poor, especially on an individual level. The establishment of the pathogenicity of ferroportin variants may not only be impeded by the presence of iron homeostasis modulating co-morbidities such as alcohol consumption and liver steatosis, but also by the limited applicability of in silico prediction models in the absence of a fully elucidated three-dimensional (3D) structure of ferroportin. In the most widely accepted secondary structure, ferroportin comprises 12 helices located in 12 transmembrane (TM) domains bound via six extracellular (ES) and five intracellular (IS) segments with a large intracellular loop between the $6^{\text {th }}$ and $7^{\text {th }}$ transmembrane helix and an intracellularly located $\mathrm{N}$ - and C-terminus. The available $3 \mathrm{D}$ models are based on a comparison with membrane transport proteins from a wide range of other species with only a $10-24 \%$ sequence homology and a maximal $40 \%$ similarity. Two studies, using E. coli glycerol-3-phosphate transporter GlpT and lactose permease LacY or the Bdellovibrio bacteriovorus Bd2019 iron transporter as template, respectively, reveal an open inward and an open outward structure with an intra- and extracellular gate between the 6 th and 7 th transmembrane domain. ${ }^{6,7}$ Site mutagenetic and conformational studies revealed the residues, located at IS1, IS2 and IS5, that are important in intracellular gate interaction and the residues, located at TM1, TM12 and ES1 and ES4, that are involved in extracellular gate interaction. These studies also identified ferroportin residues that are essential for binding and ubiquitination of hepcidin and may be involved in iron binding and egress. The unequivocally hepcidin-resistant gain-of-function ferroportin variants Cys326Ser, Tyr501Cys, Asp504Asn and Tyr64Asn and His507Arg are reported to have impaired hepcidin binding and hepcidindependent ubiquitination, respectively. In the open outward structure, variants causing impaired hepcidin binding were found to be localized within the extracellular gate, while variants causing impaired ubiquitination were found in the periphery of the molecule, suggesting that these latter variants interfere with appropriate folding after hepcidin binding. ${ }^{8}$ These findings provide a molecular basis for the observed cellular distribution pattern of the type $4 \mathrm{~B}$ iron overload in patients and the behavior in functional tests performed for these hepcidinresistant gain-of-function variants (Figure 1B).

The study by Ka et al., published in this issue, provides interesting insights into the pathophysiologic mechanisms involved in ferroportin disease caused by loss-of-function variants. They describe 22 patients from six independent families with hyperferritinemia, a normal TSAT and the heterozygous presence of the Arg178GIn variant. The serum hepcidin levels determined in two patients were above the reference range. The hepatic iron content, estimated by MRI methodology in three of the patients, was mildly elevated and a liver biopsy, performed in one patient, revealed predominant iron deposition in Kupffer cells. Although Arg178GIn displayed a reduced export of ${ }^{55} \mathrm{Fe}$ out of transfected HEK293T cells, the variant was properly localized on the cellular membrane with disappearance after 
hepcidin exposure. Based on both the clinical phenotype and functional tests the variant was assigned as a hepcidin sensitive loss-of-function variant. In the open outward conformation of the 3D structure, using Bdellovibrio bacteriovorus Bd2019 as the template, the demonstrated non-covalent interaction between Arg178 and Asp473 (located on the Nand C-lobe, respectively) was assumed to be involved in the stabilization of the open outward conformation needed to preserve iron egress. Indeed, the Asp473Ala ferroportin variant was also properly expressed on the membrane with a nearly total loss of iron export capacity. In most loss-offunction variants the abolished iron transport is attributed to defective expression of ferroportin on the membrane. Ka et al., however, provide evidence for interference in the stabilization of the conformation state, i.e., the open outward state, in the Arg178Gln variant as an alternative mechanism for diminished iron transport, despite the proper membrane expression of this variant. Further exploration of the amino-
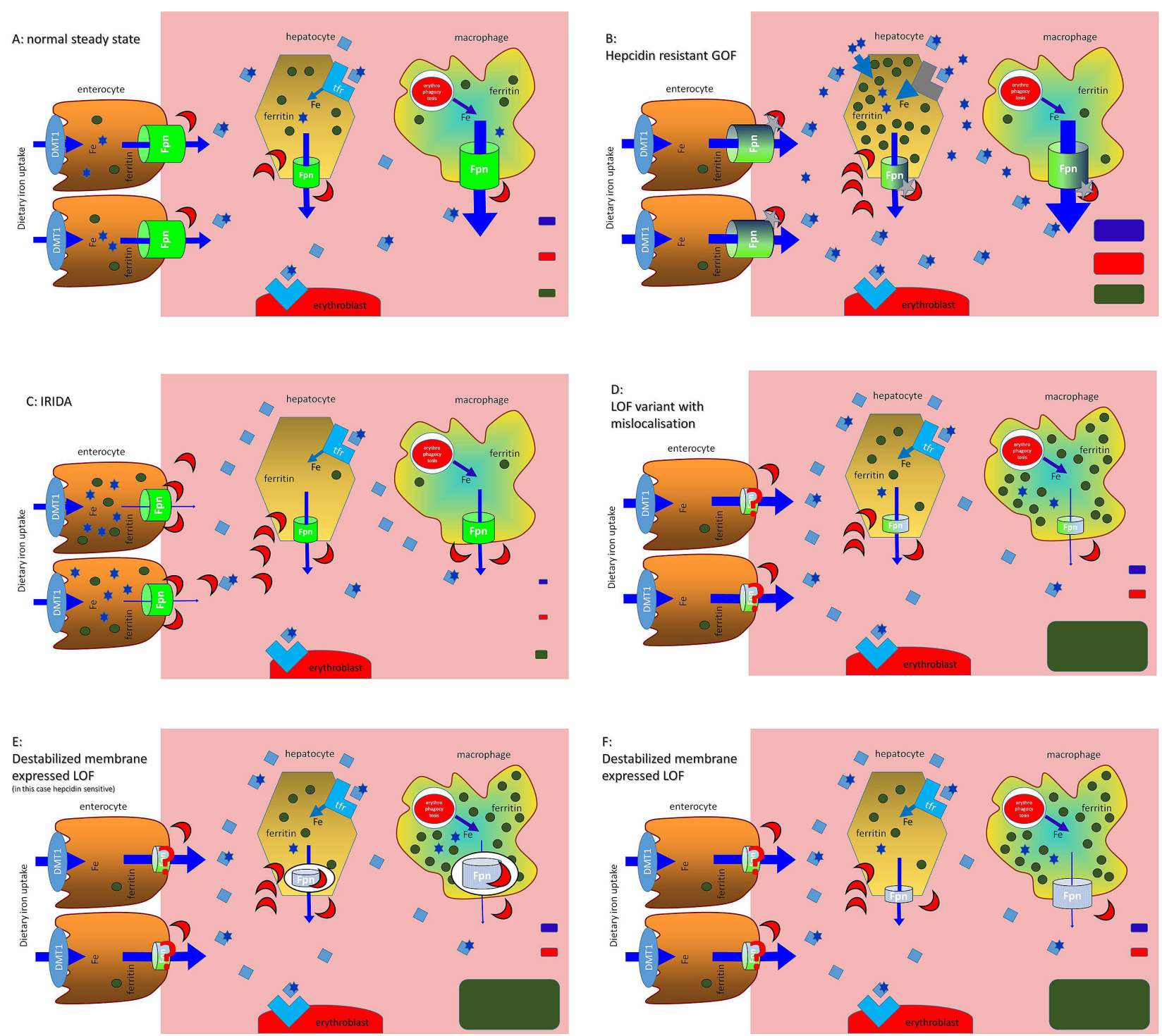

Figure 1. Model displaying cellular iron flows in patients with loss-of-function ferroportin variants as compared to physiological conditions and patients with gainof-function ferroportin variants and IRIDA. Ferroportin activity in the enterocyte, macrophage and hepatocyte in physiological steady state conditions (A), hepcidinresistant gain-of-function (GOF) (B), iron refractory iron deficiency anemia (IRIDA) (C), loss-of-function (LOF) with mislocalisation on the membrane (D), membrane expressed destabilized and hepcidin sensitive LOF (E), membrane expressed destabilized LOF (F). Physiologically, iron availability for erythropoiesis (in the form of transferrin bound iron ( ) is systemically regulated by the inhibitory effects of the hepatocyte-derived hormone hepcidin ( $A$ ) on the activity of ferroportin ( $E$ ). This regulatory system contributes to the regulation of intracellular iron ( $)$ and ferritin bound iron (O) levels, serum iron ( $\square$ ) concentration, transferrin saturation (TSAT) $(\square)$ and serum ferritin $(\square)$ concentration. In hepcidin-resistant GOF, there is an increased non-impressionable iron export out of macrophages with continuous enterocyte iron transport with iron deposition in hepatocytes. In IRIDA, ferroportin action is inhibited by (for body iron status) inappropriately elevated hepcidin leading to decreased enterocyte iron resorption and diminished ferric-transferrin availability for the erythroblast with the sequestering of some iron, derived from erythrophagocytosis, within the macrophage. In patients with LOF variants there is decreased iron transport out of the macrophage leading to iron sequestration in these cells, but the mechanism of the apparently relatively increased enterocyte iron export with subsequent body iron overload is not yet fully elucidated. Clinically observed changes in serum iron, and ferritin concentration and TSAT in the various conditions are depicted by the size of the rectangles in the lower right-hand side of the panels. DMT: divalent metal transporter; tfr: transferrin receptor-1; fe: iron. Transferrin ( $\square$ ). 
acid interaction network in various ferroportin conformation states may reveal additional sites essential for stabilization and provide molecular explanation for defective iron egress in variants without defective membrane expression, such as Arg88Gly, Leu129Pro, Ile152Phe and Asn174Ile..$^{9-11}$

Notwithstanding the importance of this finding, it does not clarify the iron overload observed in these patients. Moreover, as reported by $\mathrm{Ka}$ and colleagues, it is notable that the patients with a loss-of-function variant ferroportin, that completely disappears from the cellular membrane after exposure to hepcidin, have no anemia and intensive phlebotomy regimens were reported to be well tolerated. In patients with defective iron export transport due to a lossof-function variant, either due to mislocalization or improper folding, one might expect diminished enterocyte iron absorption leading to a phenotype similar to that described for iron-refractory iron deficiency (IRIDA). In IRIDA, mutations in the TMPRSS6 gene result in inappropriately elevated serum hepcidin levels with diminished ferroportin activity and a variable degree of iron deficiency anemia, unresponsive to oral iron treatment, and intra-enterocyte iron retention in experimental animals (Figure 1C). ${ }^{12,13}$ Experimental animals with diminished ferroportin activity due to monoallelic wild-type ferroportin expression also display iron deficiency anemia. ${ }^{2}$ This is in contrast to the situation in patients with diminished ferroportin activity due to loss-of-function ferroportin variants which is characterized by iron overload, normal circulating hemoglobin concentrations and absence of intra-enterocyte iron (evaluated in a limited number of patients) (Figure 1D,E). ${ }^{14}$ It appears that in the enterocyte diminished ferroportin activity due to decreased activity of wild-type ferroportin has a different effect on iron export capacity than decreased ferroportin activity caused by a loss-of-function variant. In addition, the impact of loss-of-function variants on iron export capacity may be different between macrophages and enterocytes, and the results of the functional studies in HEK293T cells are more predictive for macrophage iron handling. It has been suggested that in patients with lossof-function variants the remaining monoallelic expressed wild-type ferroportin protein would be sufficient to preserve iron export in cells with low iron turnover, such as enterocytes, but insufficient to maintain iron export capacity in cells with high iron turnover, such as macrophages, with subsequent intracellular iron retention in these cells. ${ }^{5}$ However, this theory may not provide a full explanation for the state of iron overload in these patients. While the activity of ferroportin in both enterocytes and macrophages in the systemic regulation of iron homeostasis is mediated by hepcidin, there is a considerable amount of experimental data indicating that the regulation of ferroportin expression and activity differ between enterocytes and macrophages. These data include differences in intracellular regulatory mechanisms that fine-tune ferroportin membrane expression as well as differences in ferroportin activity upon various systemic stimuli. ${ }^{15-20}$ The study by $\mathrm{Ka}$ et al. contributes to our understanding of the pathogenic mechanisms involved in decreased ferroportin activity by loss-of-function variants. Studies focused on the consequences of these variants on enterocyte iron handling are warranted to further comprehend the pathophysiology of this intriguing iron overload disorder.

\section{References}

1. Abboud S, Haile DJ. A novel mammalian iron-regulated protein involved in intracellular iron metabolism. J Biol Chem. 2000;275 (26):19906-19912.

2. Donovan A, Lima CA, Pinkus JL, et al. The iron exporter ferroportin/Slc40a1 is essential for iron homeostasis. Cell Metab. 2005;1(3):191-200.

3. Ganz T, Nemeth E. Hepcidin and iron homeostasis. Biochim Biophys Acta. 2012;1823(9):1434-1443.

4. Nemeth E, Tuttle MS, Powelson J, et al. Hepcidin regulates cellular iron efflux by binding to ferroportin and inducing its internalization. Science. 2004;306(5704):2090-2093

5. Pietrangelo A. The ferroportin disease: pathogenesis, diagnosis and treatment. Haematologica. 2017;102(12):1972-1984.

6. Bonaccorsi di Patti MC, Polticelli F, Cece G, et al. A structural model of human ferroportin and of its iron binding site. FEBS J. 2014;281 (12):2851-2860.

7. Taniguchi R, Kato HE, Font J, et al. Outward- and inward-facing structures of a putative bacterial transition-metal transporter with homology to ferroportin. Nat Commun. 2015;6:8545.

8. Aschemeyer S, Qiao B, Stefanova D, et al. Structure-function analysis of ferroportin defines the binding site and an alternative mechanism of action of hepcidin. Blood. 2018;131(8):899-910.

9. Moreno-Carralero MI, Munoz-Munoz JA, Cuadrado-Grande N, et al. A novel mutation in the SLC40A1 gene associated with reduced iron export in vitro. Am J Hematol. 2014;89(7):689-694.

10. Le Gac G, Ka C, Joubrel R, et al. Structure-function analysis of the human ferroportin iron exporter (SLC40A1): effect of hemochromatosis type 4 disease mutations and identification of critical residues. Hum Mutat. 2013;34(10):1371-1380.

11. Callebaut I, Joubrel R, Pissard S, et al. Comprehensive functional annotation of 18 missense mutations found in suspected hemochromatosis type 4 patients. Hum Mol Genet. 2014;23(17):4479-4490.

12. De Falco L, Sanchez M, Silvestri L, et al. Iron refractory iron deficiency anemia. Haematologica. 2013;98(6):845-853.

13. Folgueras AR, de Lara FM, Pendas AM, et al. Membrane-bound serine protease matriptase-2 (Tmprss6) is an essential regulator of iron homeostasis. Blood. 2008;112(6):2539-2545.

14. Corradini E, Montosi G, Ferrara F, et al. Lack of enterocyte iron accumulation in the ferroportin disease. Blood Cells Mol Dis. 2005;35(3):315-318.

15. Zhang DL, Ghosh MC, Rouault TA. The physiological functions of iron regulatory proteins in iron homeostasis - an update. Front Pharmacol. 2014;5:124

16. Drakesmith $\mathrm{H}$, Nemeth $\mathrm{E}$, Ganz T. Ironing out ferroportin. Cell Metab. 2015;22(5):777-787.

17. Canonne-Hergaux F, Donovan A, Delaby C, et al. Comparative studies of duodenal and macrophage ferroportin proteins. Am J Physiol Gastrointest Liver Physiol. 2006;290(1):G156-163.

18. Theurl I, Aigner E, Theurl M, et al. Regulation of iron homeostasis in anemia of chronic disease and iron deficiency anemia: diagnostic and therapeutic implications. Blood. 2009;113(21):5277-5286.

19. Jacolot S, Ferec C, Mura C. Iron responses in hepatic, intestinal and macrophage/monocyte cell lines under different culture conditions. Blood Cells Mol Dis. 2008;41(1):100-108.

20. Chaston T, Chung B, Mascarenhas M, et al. Evidence for differential effects of hepcidin in macrophages and intestinal epithelial cells. Gut. 2008;57(3):374-382. 